\title{
La Voce dei Pazienti Essere in dialisi: molta fatica, molti preziosi rapporti umani
}

Per qualcuno è una grossa sofferenza, mentre, per altri, è un'esperienza della vita che fa incontrare e conoscere persone come loro, che soffrono ma che tengono duro e vanno avanti. E possono nascere amicizie e legami molto forti, che aiutano ad affrontare questa prova con maggiore serenità.

S.B.: Mia mamma, molto probabilmente, dovrà iniziare la dialisi. Volevo chiedere a chi sta già vivendo questa esperienza quanto cambia la vita quotidiana, perché mia mamma è terrorizzata. Grazie fin d'ora a tutti.

G.P.: Iniziare la dialisi è come nascere una seconda volta e, come per ogni nascita, c'è bisogno di un periodo di adattamento... Le grandi difficoltà e il conseguente rifiuto al trattamento riguardano le molteplici rinunce in campo sociale e alimentare che il paziente deve sopportare. Quello che posso consigliarvi è sostegno psicologico e fisico, soprattutto dopo le sedute di emodialisi, quando la pressione scende tanto che sembra quasi di morire... Non solo sopportate i suoi cambi di umore, ma, allo stesso tempo, spronatela in attività differenti, come passeggiate e hobby vari. Da figlia di una dializzata e io stessa futura dializzata mando a te e a tua madre un abbraccio fortissimo!

M.G.: Ricordi di tanti anni fa. La grande stanchezza dopo le quattro ore di dialisi un giorno sì e uno no. Non ero più in grado di gestire la casa. Mia figlia aveva 12 anni. Mio marito doveva fare le pulizie e cucinare. Stai vicino a tua mamma nell'aiuto domestico, portale un libro divertente... Un abbraccio!

A.F.: Quindi, state dicendo che facendo dialisi si sta male?

G.P.: Si può soffrire di ipotensione e stanchezza, ma non è uguale per tutti... Molto è correlato al peso.

P.G.: Ciao. Io non sono ancora in dialisi, ma mio papà I'ha fatta per dieci anni e mia mamma per sei mesi. Mio padre l'ha presa come una vera salvezza e soluzione ai vari problemi di alimentazione. È sempre stato bene, anche dopo la terapia, e, al massimo, dopo pranzo faceva un riposino. La vedeva come una cosa da fare per vivere, perché, senza, non ci sarebbe più stata vita. Voleva bene alla sua macchina al reparto e a tutti gli operatori. Non ha mai detto una parola negativa e, a volte, penso che non l'abbia fatto per non spaventarmi. Per mia mamma è stato solo un periodo transitorio. Poi ha smesso. Non so come la prenderò quando toccherà a me, ma mi ricorderò sicuramente la tranquillità con cui la affrontava il mio papà!
A.M.: Non drammatizziamo. La dialisi non va demonizzata. È come la policistosi: varia da individuo a individuo. Va affrontata come una tappa di transito, in attesa del trapianto. Il cambiamento è, senz'altro, positivo, si passa da uno stato di tossicità alla libertà di mangiare senza sacrifici, riducendo solo l'apporto di liquidi. A tutto ci si abitua, anche alla dialisi. L'importante è affrontarla con serenità e determinazione. Le quattro ore non passano mai, se ci si piange addosso. Non te ne accorgi se leggi, guardi la tv o parli con gli infermieri. Saranno loro per primi i tuoi amici e altri pazienti che, magari, soffrono più di te. Insomma, si acquisisce un'altra famiglia. Per me è stata un'esperienza notevole dal punto di vista dei rapporti umani, tanto che, anche dopo il trapianto, continuo a frequentare il mio centro dialisi. Faccio gli auguri a tutti coloro che sono o che stanno per entrare in dialisi, dicendo loro: non abbiate alcuna preoccupazione. È solo un'altra tappa della nostra vita.

G.C.: Caro A.M., il tuo modo di affrontare le cose è davvero eccezionale. Mi ricordi tantissimo il mio papà e come lui ha affrontato i 14 anni di dialisi. I suoi amici sono diventati i nostri amici, i suoi compagni di viaggio i nostri compagni dei pranzi della domenica. Uniti a condividere il male, ma anche il bene, di quell'esperienza. Non sono stati anni facili, mio papà spesso tornava a casa con la pressione ai minimi storici. E gli sbalzi d'umore c'erano. Ma la dialisi gli ha permesso di restare con noi e di rafforzare i rapporti umani, come dici tu. Ti auguro che la vita ti dia il meglio, ricompensando tutta la positività che metti nelle cose.

A.M.: Mio padre è stato in dialisi per 6 anni e mezzo. II prossimo Dicembre saranno passati tre anni dal trapianto. lo sono in dialisi da due mesi e mezzo. A parte qualche livido fin quando le vene non si sviluppano, la macchina diventa la tua amica e la tua salvezza. II mio $46^{\circ}$ compleanno l'ho festeggiato con l'equipe e i pazienti, alcuni dei quali facevano dialisi già con mio padre... Per me è un po' pesante la gestione familiare, perché mia figlia ha quasi 13 anni, però ho più forza rispetto all'ultimo anno. Allora non vivevo affatto, ora, invece, faccio più cose e, quando sono stanchissima, dormo. Puoi leggere, guardare la tv o ascoltare musica con il cellulare... lo mi vizio con caramelline 
al miele e un pacchetto di taralli... Stai serena...

S.F.: Grazie, A.M., per le tue parole. Mio padre ha quasi 78 anni, da cinque fa dialisi peritoneale e dallo scorso Natale non è stato praticamente mai bene, compresa la depressione. Sono stati mesi allucinanti, con mia mamma sempre al suo fianco. Ora sta iniziando l'extracorporea una volta a settimana e, per il resto, ancora peritoneale. Vorrei tanto che avesse il tuo spirito, come lo vorrebbe anche il resto dei familiari. Non credo possa sperare in un trapianto. Lui è totalmente diverso dall'uomo battagliero che conoscevo e i miei familiari stanno sempre a pensare al peggio. Speriamo bene. Bello che ci siano persone come te.
L.P.: La mia mamma ha fatto dialisi per 26 anni ed è stata sempre, come la chiamavo io, una forza della natura: non ha mai rinunciato a godersi la vita per nessuna ragione al mondo, era sempre con il sorriso sulle labbra e mi diceva sempre "pazienza, l'importante è essere con voi".

C.D.: Faccio la dialisi da quattro mesi e anche io ero terrorizzata, ma, quando la mia vita è diventata un incubo ed ero a un passo dal coma, mi sono decisa. Ora sono contenta, perché sto bene.

Published online: August 3, 2016 